Hospice is carrying out further work to establish whether this will support more patients to achieve their preferred place of final care.

Evaluation by Worcester University demonstrates communities willingness to engage with service providers and commissioners.

\title{
P11 COMPASSIONATE COMMUNITIES: FROM REACHING PEOPLE TO WORKING IN PARTNERSHIP WITH COMMUNITIES AT EOL
}

Diana Murungu, Daphne Welch, Tina Swani Birmingham St. Mary's Hospice, Birmingham, UK

10.1136/bmjspcare-2011-000105.11

Introduction Birmingham St. Mary's Hospice (BSMH) is working to increase access to excellent EoLC services to patients and their families from minority communities in Birmingham. Through this work we are aware that communities provide support to their members at end of life and during bereavement. Communities appear to take their contribution for granted as their 'way of life.'

Aims Increase awareness and use of palliative care services for minority communities. Increase conversations around death, dying and bereavement. Identify and work with compassionate communities to support patients and families in the community through partnership working with clinicians. Create a forum for sharing care ideas between communities and service providers.

\section{Methods}

- Qualitative methods: Purposive and snowballing sampling. Education for clinicians at the hospice.

- 'Sharing Care' Conference.

Results Compassionate communities exist in Birmingham who support their own members at end of life. They are willing to work in partnership with clinicians to support patients. A Reference group made up of community members has been set up at the Hospice. Community leaders participate in our training programme for clinicians to raise awareness of how culture and spirituality can impact on access to services.

Discussion points The extent of commitment to partner communities by service providers is still to be tested. The 